\title{
Quantitative clinical characteristics of 53 patients with MPS VII: a cross-sectional analysis
}

\author{
Matthias Zielonka, MD1', Sven F. Garbade, PhD'1, Stefan Kölker, MD', Georg F. Hoffmann, MD¹ \\ and Markus Ries, MD, PhD ${ }^{1}$
}

Purpose: The main purpose of the study was to provide quantitative data regarding survival and diagnostic delay. Mucopolysaccharidosis (MPS) type VII (OMIM 253220) is a progressive neurometabolic disorder caused by deficiency of the lysosomal enzyme $\beta$-glucuronidase (GUS). Hard clinical end points have not been quantitated

Methods: We quantitatively analyzed published cases with MPS VII $(N=53 / 88$ with sufficient data). Main outcome measures were onset of disease and survival. The role of biomarkers such as GUS residual enzyme activity and levels of storage material assessed as urinary excretion of glucosaminoglycans (GAG) as potential predictors of clinical outcomes were investigated. The analysis was conducted according to STROBE criteria.

Results: Median survival of the postnatally diagnosed population was 42 months. Median age of disease onset was the first day of life; median age at diagnosis was 11 months. Hydrops fetalis was frequent. Patients with residual GUS activity in fibroblasts more than $1.4 \%$ or urinary GAG excretion less than $602 \%$ of normal survived longer than patients with GUS enzyme activity below or GAG excretion above these thresholds.

Conclusion: MPS VII has its disease onset prenatally. In the absence of a prenatal diagnosis, most cases are clinically apparent at birth. Our data corroborate a phenotype-biomarker association in MPS VII. The survival data characterize the natural history with important implications for therapeutic studies.

Genet Med advance online publication 6 April 2017

Key Words: drug development; mucopolysaccharidosis; natural history; orphan disease; Sly disease

\section{INTRODUCTION}

Mucopolysaccharidosis type VII, also known as Sly disease (OMIM 253220), is a progressive neurometabolic disorder caused by inherited deficiency of the lysosomal enzyme $\beta$-glucuronidase. The disorder leads to the progressive storage of the glucosaminoglycans dermatan sulfate, heparan sulfate, and chondroitin 4-,6-sulfate in lysosomes of various tissues. ${ }^{1}$ The cytogenetic location of GUSB, the gene encoding $\beta$-glucuronidase, is $7 \mathrm{q} 11.21 .^{2}$ Fifty-four different mutations are currently known, of which 43 are missense/nonsense, 5 are splicing, 1 is a regulatory mutation, 4 are small deletions, and 1 is a gross deletion. ${ }^{3}$ The estimated prevalence is 0.01 in 100,000 live births. ${ }^{4}$ On 16 February 2012, recombinant human $\beta$-glucuronidase received orphan drug designation by the US Food and Drug Administration and is currently (as of 17 August 2016) the only compound in development for the treatment of mucopolysaccharidosis (MPS) VII. ${ }^{5}$ Clinical trials involving recombinant $\beta$-glucuronidase are ongoing (phase I/II study NCT01856218, phase III study NCT02230566).

In a recent interview-based multicenter survey of 56 patients, neonatal nonimmune hydrops fetalis was frequently observed; other common clinical manifestations were short stature, skeletal dysplasia, hepatosplenomegaly, hernias, cardiac involvement, pulmonary insufficiency, and cognitive impairment. ${ }^{6}$
Quantitative data regarding survival of MPS VII are not available. ${ }^{7}$ Survival is important for counseling parents. In addition, reliable data about the survival of untreated patients as an important hard end point in clinical trials would be indispensable to assessing the long-term impact of potential novel therapies. In addition, MPS VII is an ultrarare disorder that renders the diagnostic suspicion difficult. The precise diagnostic delay is not known. With the development of novel therapies, it would be important to know whether there is a need to raise awareness for this disorder to accelerate the diagnostic process. Although there is a plethora of published cases or case series in the medical literature that exceeds, by far, the number of patients included in the survey by Montaño and colleagues, ${ }^{6}$ a systematic quantitative analysis of all published cases has never been undertaken. Such an approach is of value because the rare nature of the disease renders unfeasible the prospective natural history studies with large numbers of patients. Because MPS VII is a rare disorder, and establishing the diagnosis requires assays that are not available in a routine care setting, there is increased risk of considerable diagnostic delay and missing patients due to incorrect diagnosis. Disease awareness becomes important as soon as a specific therapy becomes available, taking into account that there may be a limited window of opportunity for early intervention.

${ }^{1}$ Division of Pediatric Neurology and Metabolic Medicine, Center for Pediatric and Adolescent Medicine, University Hospital Heidelberg, Heidelberg, Germany. Correspondence: Markus Ries (markus.ries@uni-heidelberg.de) 


\section{ORIGINAL RESEARCH ARTICLE}

The purpose of this study was to provide quantitative data regarding survival and diagnostic delay. In addition, we hypothesized that the presence of residual enzyme activity is associated with delayed onset of clinical disease and prolonged survival of afflicted individuals.

\section{MATERIALS AND METHODS}

The analysis was performed according to STROBE (Strengthening the Reporting of Observational Studies in Epidemiology) criteria (http://www.strobe-statement.org).

\section{Literature review and definitions of variables}

We conducted an exhaustive literature search on PubMed with the keywords "mucopolysaccharidosis type 7," "Sly disease," and "beta-glucuronidase (GUS) deficiency." All publications were downloaded $(N=359)$ and manually sorted for reports containing clinical, biochemical, and genetic information $(N=74)$. Reports were published between 1973 and 2015. There were sufficient data for analysis for 88 patients (from 70 publications) (Figure 1). The articles were published in English, French, and Spanish. The close of database was 15 October 2015. None of the analyzed patients received enzyme replacement.

The following variables were extracted: age at onset and at diagnosis; mode of diagnosis; symptoms leading to the diagnosis; last reported age; information regarding whether the patient is alive or deceased; $\beta$-glucuronidase activity in plasma, leukocytes, fibroblasts, amniocytes or chorionic villus cells; and excretion of glucosaminoglycans (GAG) in urine.

If information regarding time was provided in semiquantitative terms in the respective publications, we took a conservative approach and defined the findings as follows: "stillborn," day 0; "newborn" or "at birth," day 1; "newborn period," 1 month; and "postmortem," age at death.

\section{Statistical analysis}

Techniques of descriptive statistics were applied in a manner similar to that reported previously. ${ }^{8}$ Variables were summarized using counts and percentages of the total population. Because biochemical parameters (enzyme activity, glycosaminoglycan levels) were assessed in different laboratories, values were expressed as a percentage of the mean of the reported normal range. Survival was calculated as the time between patient birth and time of death. Patient data were censored at the time of last follow-up if the patient was reported to be alive at the time of the last visit. Survival was estimated with the Kaplan-Meier method. The log-rank test was applied to compare potential differences in survival between subgroups. Unbiased recursive partitioning was used to determine cutoff values for residual GUS activities and urinary GAG excretion in sub-group analyses. ${ }^{9}$ Time to diagnosis for a given patient was calculated as the difference between age at diagnosis and the age at onset. Missing data were not imputed. Sensitivity analyses were not conducted. All analyses were performed using R (http://www.rproject.org). $P$ values reported were two-sided. $P \leq 0.05$ was considered statistically significant.

\begin{abstract}
RESULTS
Overall, we identified 67 of 88 patients (from articles published between 1973 and 2015) for further statistical analysis. Of those, 53 patients (articles published between 1973 and 2012) were diagnosed postnatally; for the remaining 14 , the diagnosis of MPS VII had already been established prenatally. Demographic characteristics of the postnatally diagnosed cohort are shown in Table 1. For different subsequent data analyses, patients had to be excluded due to missing data in the observed subsets. Sample sizes $(N)$ are always indicated for the corresponding analyses.
\end{abstract}

\section{Prenatally diagnosed patients}

Fourteen patients were diagnosed prenatally. Of those, nine pregnancies were terminated and five patients died during a later course of pregnancy or at birth. The longest survival of the prenatally diagnosed patients was 1 day. Three patients were identified by selective testing due to a prior affected sibling. The remaining 11 patients were diagnosed because they exhibited prenatal features suggestive of MPS VII.

\section{Postnatally diagnosed patients}

Median age of onset of disease in the group of postnatally diagnosed patients was 0.03 months (i.e., the first day of life; IQR, 1 day to 1 month; $N=50$ ). Median age at diagnosis was 11 months (IQR, 1 to 60 months; $N=53$ ). Median diagnostic delay was 9.99 months (IQR, 1.3 to 47.7 months; $N=50$ ) (Figure 2).

The median estimated survival for the group of the postnatally diagnosed patients $(N=53)$ was 42 months (Figure 3a). There was no difference in survival between male and female patients (log rank test, $P=0.50$ ). Patients with residual GUS enzyme activity $>1.4 \%$ in fibroblasts had longer survival compared with patients whose residual enzyme activity in fibroblasts was $\leq 1.4 \%$ of normal $(P<0.01$, log-rank test; $N=27$; threshold determined by unbiased recursive partitioning) (Figure 3b). In addition, simple linear regression indicated that higher residual enzymatic activity was associated with later onset of the disease but only relies on a limited number of patients. Patients with glucosaminoglycan levels $<602 \%$ of normal, with the threshold determined by unbiased recursive partitioning, had longer survival compared with patients whose glucosaminoglycan levels were $>602 \%$ of normal $(P<0.05$, log-rank test) (Figure 3c). By contrast, higher GAG excretion was not associated with an earlier age of onset. Furthermore, residual $\beta$-glucuronidase activity in plasma or leukocytes did not correlate with age of onset or survival. Descriptive statistical values are depicted in Table 2. We analyzed patient survival rates published before and after 1990 (threshold determined by median split). There was no significant difference in survival before and after $1990(P=0.27$, log-rank test).

We analyzed the distribution of presenting or leading signs or symptoms in the study population (Supplementary Table S3 online). The most common symptoms were skeletal deformities, hydrops fetalis, hepatosplenomegaly, hernias, and coarse facies, as well as mental retardation or disability. Hydrops fetalis as the very first clinical symptom of MPS VII was observed in 
Publications on Pubmed published until October 2015 using "Mucopolysaccharidosis type VII", "Sly disease" or "beta-glucuronidase deficiency" as search term

$$
n=359
$$

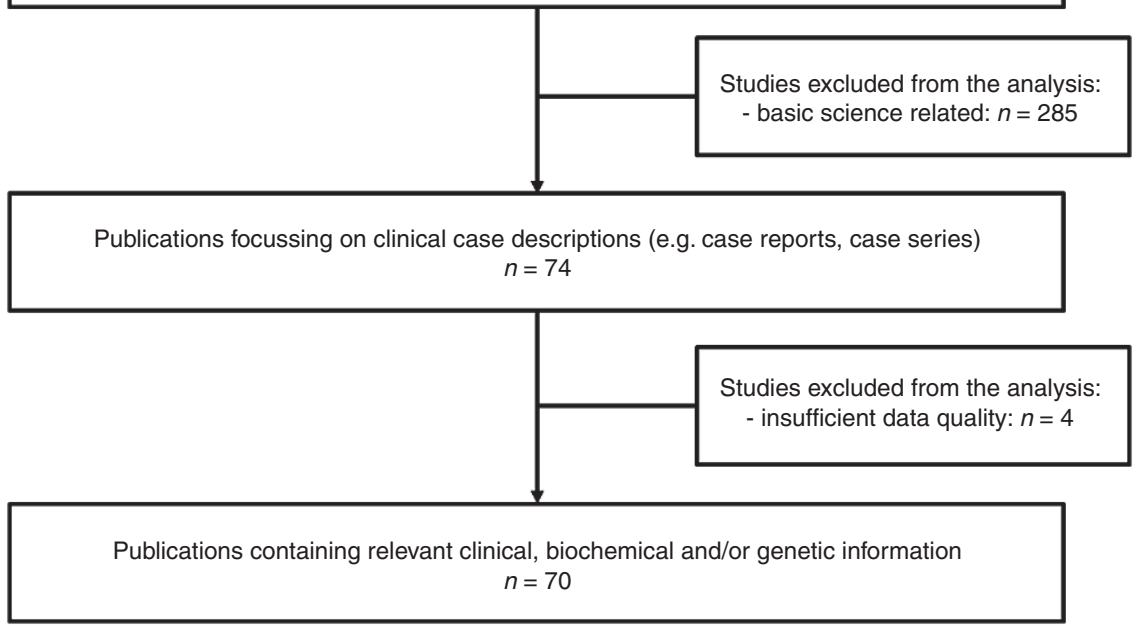

Figure 1 Identification of clinical case reports and case series of patients with mucopolysaccharidosis type VII. The reports analyzed were published on PubMed up to 15 October 2015. Study flow diagram.

Table 1 Demographics of the study population with postnatally diagnosed mucopolysaccharidosis type VII $(N=53)$

\begin{tabular}{lcc} 
Population characteristics & N & $\%$ \\
\hline Sex & & \\
Female & 22 & 41.5 \\
Male & 30 & 56.6 \\
Unknown & 1 & 1.9 \\
Mode of diagnosis & & \\
• Enzymatic & 33 & 62.3 \\
• Genetic & 1 & 1.9 \\
• Enzymatic and genetic & 19 & 35.8 \\
Alive at last follow-up & & \\
Yes & 33 & 62.3 \\
No & 20 & 37.7 \\
\hline
\end{tabular}

$45.5 \%$ of cases (Supplementary Table S3 online) in both prenatally and postnatally diagnosed patients.

\section{DISCUSSION}

We have quantitatively defined age of onset, diagnostic delay, and survival of patients with MPS VII. In addition, biomarkers were correlated with the clinical outcome in the natural history. Higher residual $\beta$-glucuronidase enzyme activity in fibroblasts and lower GAG excretions in urine were associated with longer survival.

The most common signs and symptoms were skeletal deformities, hydrops fetalis, hepatosplenomegaly, hernias, coarse facies, and cognitive impairment. Although this was not a primary focus of the present study owing to the inherent methodological challenges of extracting information regarding soft end points from published results, these data corroborate the interview-based study by Montaño and colleagues. ${ }^{6}$ Compared

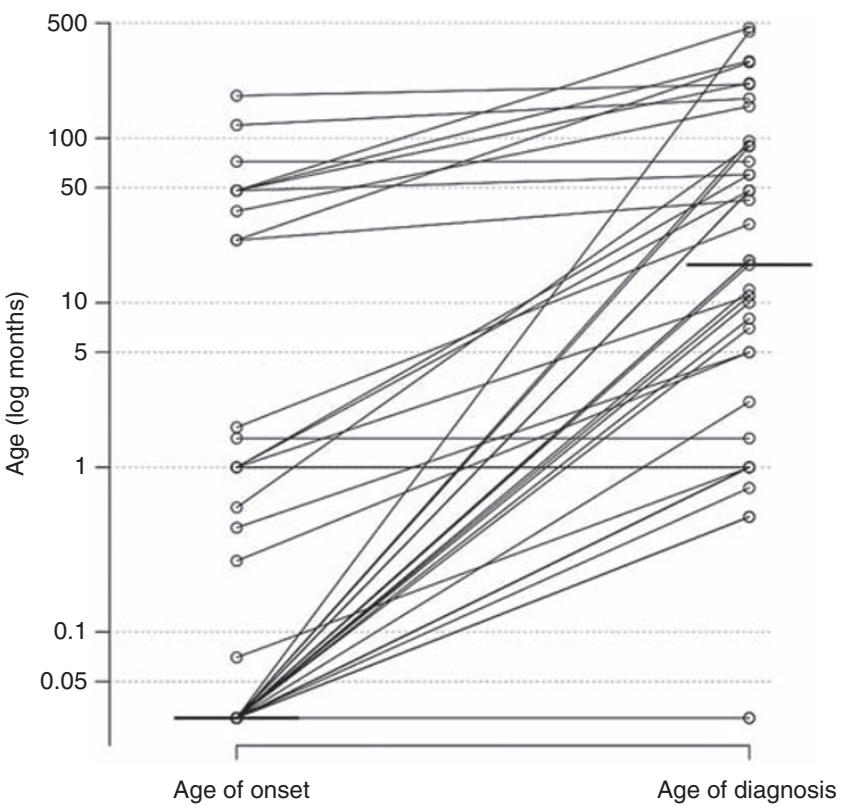

Figure 2 Age at onset (in months) of mucopolysaccharidosis type VII and age at diagnosis. Data were available for 50 patients. Horizontal lines indicate the median. The slopes of connecting lines represent the diagnostic delay between onset of the disease and the time of diagnosis.

to the interview-based study, overall underrepresentation of most of the clinical signs and symptoms in our cohort is most likely due to the lack of a standardized protocol used by the reporting physicians. Furthermore, data quality of soft end points was based predominantly on clinical descriptions performed by physicians in different specialist fields, which might also contribute to the differences.

Lysosomal storage diseases are an important differential diagnosis in the workup of nonimmune hydrops fetalis. Although cardiovascular disorders (30\%), extracardiac anomalies (20\%), 

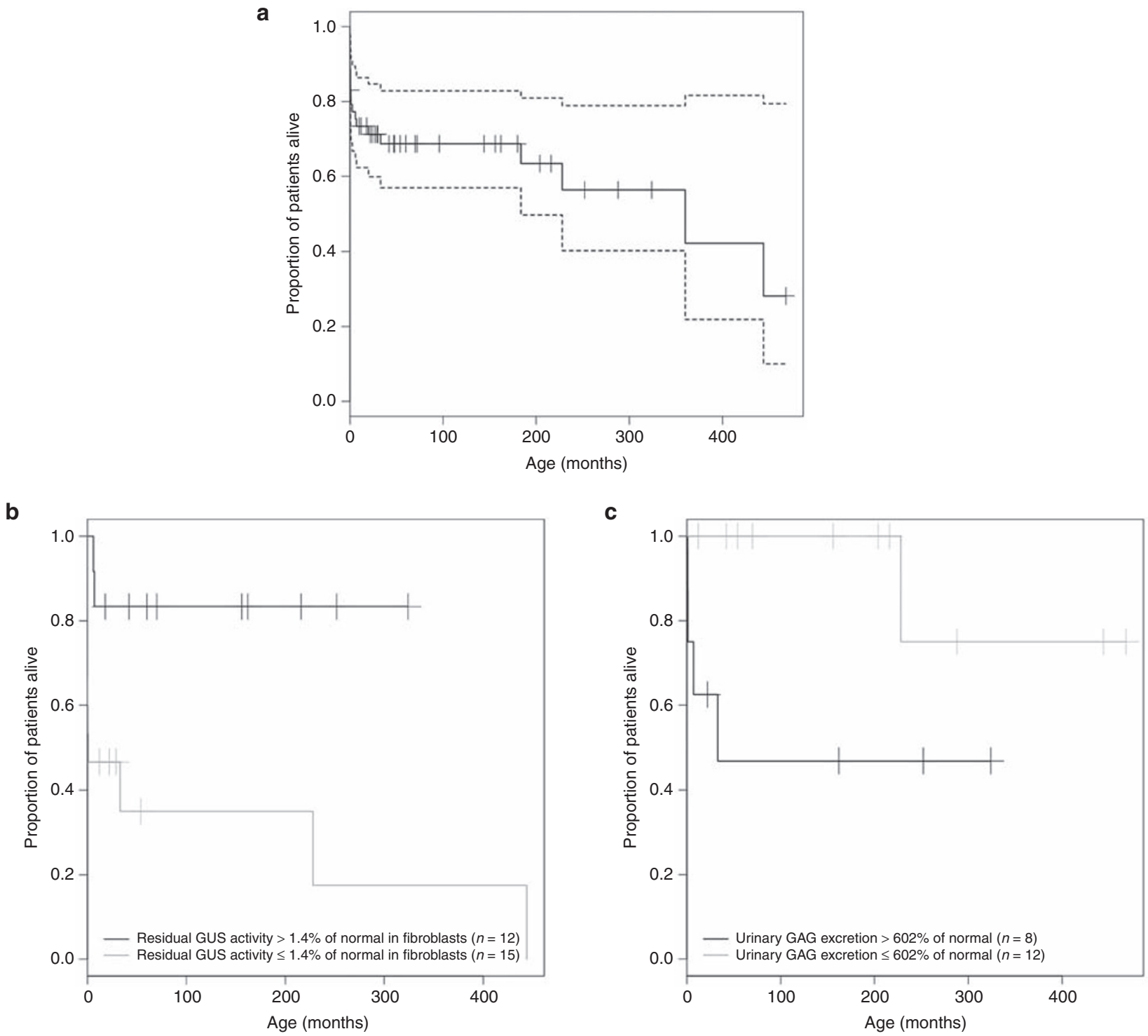

Figure 3 Estimated survival distributions for postnatally diagnosed patients with mucopolysaccharidosis type VII (MPS VII). (a) Estimated overall survival distribution for MPS VII patients $(N=53)$. Censored individuals are marked with " + ". (b) Estimated survival distribution for MPS VII patients with residual GUS enzyme activity $>1.4 \%(N=12$, thick line) and $\leq 1.4 \%$ of normal controls ( $N=15$, thin line). Enzymatic activity was measured in fibroblasts. Censored individuals are marked with " + ". Log-rank test, $P=0.0077$. (c) Estimated survival distribution for MPS VII patients with urinary GAG excretion $>602 \%(N=8$, thick line) and $\leq 602 \%$ of normal controls $(N=12$, thin line). Censored individuals are marked with " + ". Log-rank test, $P=0.0374$. The GAG excretion threshold was determined via unbiased recursive partitioning.

Table 2 Biochemical, enzymatic, and survival characteristics of the study population with prenatally and postnatally diagnosed mucopolysaccharidosis type VII

\begin{tabular}{|c|c|c|c|c|c|c|}
\hline Variable & $N$ & Mean \pm SD & Median & IQR & Min. & Max. \\
\hline \multicolumn{7}{|c|}{ Residual GUS activity (\% of mean of normal control) } \\
\hline - Serum & 22 & $1.2 \pm 1.97$ & 0.55 & $0.18-1.23$ & 0 & 7.1 \\
\hline - Leukocytes & 25 & $1.46 \pm 1.7$ & 1.4 & $0.28-1.85$ & 0 & 8.4 \\
\hline - Fibroblasts & 37 & $2.45 \pm 3.75$ & 1.4 & $0.30-2.15$ & 0 & 18.4 \\
\hline \multicolumn{7}{|c|}{ GAG excretion in urine (\% of mean of normal control) } \\
\hline - > Cutoff of $602 \%$ of normal & 8 & $1,224 \pm 809$ & 905 & $721-1,332$ & 608 & 3,000 \\
\hline - $<$ Cutoff of $602 \%$ of normal & 12 & $257 \pm 178$ & 186 & $124-354$ & 100 & 602 \\
\hline \multicolumn{7}{|l|}{ Last reported age (months) } \\
\hline - Prenatally diagnosed & 5 & $0.006 \pm 0.013$ & 0.00 & $0.00-0.00$ & 0.00 & 0.03 \\
\hline - Postnatally diagnosed & 53 & $108.14 \pm 135.22$ & 44.50 & $5.25-182.7$ & 0.00 & 468.0 \\
\hline
\end{tabular}


chromosomal disturbances (20\%), infections (10\%), hematologic anomalies (5-10\%), and monochorionic twin pregnancies (5-10\%) are common underlying causes of nonimmune hydrops fetalis, lysosomal storage diseases have been reported to account for 1 to $15 \%$ of the cases associated with this condition, with the most frequent being mucopolysaccharidosis type VII, Gaucher disease, $\mathrm{G}_{\mathrm{M} 1}$-gangliosidos, and sialidosis. ${ }^{10}$ In accordance with these previously published data, half of the MPS VII patients investigated (45.5\%) demonstrated hydrops fetalis as the very first clinical sign, emphasizing the fact that MPS VII should be considered in any case of unexplained hydrops fetalis as soon as red blood cell alloimmunization has been ruled out.

Currently, the two most important major barriers to success in treating MPS VII are (i) translation of preclinical results into cases and (ii) early diagnosis within the yet-to-be defined "therapeutic window of opportunity" of reversibility and prevention of morbidity. Various therapeutic approaches were tested in murine or canine animal models with encouraging results, such as enzyme replacement, ${ }^{11,12}$ stem cell transplantation, ${ }^{13}$ and gene therapy. ${ }^{14-16}$ A 12-year-old boy with MPS VII was experimentally treated for a short time course ( 24 weeks) with recombinant human GUS. The treatment was well tolerated, GAG levels declined, and liver and spleen sizes normalized. ${ }^{17}$ If these encouraging results are confirmed in larger human studies, then late diagnosis is still a major barrier to successful treatment, because the onset of disease is prenatal and evident in the majority of patients on the first day of life. Currently, the diagnostic delay is also substantial, as illustrated by our findings. Early diagnosis is important, as in many diseases; early therapy may lead to better outcomes, which could also have strategic implications for newborn screening. This emphasizes the importance of disease awareness for MPS VII. As of 30 September 2016, five open clinical studies were registered at ClinicalTrials.gov (http://www.clinicaltrials.gov). Because a relevant subset of patients has a severe form of hydrops fetalis that manifests prenatally, it may become necessary for intrauterine treatment to be considered. Our data regarding diagnostic delay will serve as a baseline for future diagnostic performance.

Our data document a phenotype-biomarker association in MPS VII. These findings are in line with the threshold theory of residual enzyme activity. In lysosomal storage disorders, there is an extremely high degree of phenotypic heterogeneity, i.e., a large spectrum of age of onset and clinical severity. Although many of these disorders have been classified into subforms of types, such as juvenile vs. adult, early onset vs. late onset, classic vs. variant, mild vs. severe, and neuronopathic vs. nonneuronopathic, this heterogeneity is a continuous spectrum. ${ }^{18}$ Frequently, there is an overlap between these subtypes. ${ }^{19,20}$ Conzelmann and Sandhoff proposed the threshold theory of residual enzyme activity to explain the correlation between severity of the disease and residual enzyme activity. The model of residual enzyme activity is based on the assumption that the mutated catabolic enzyme has not lost its catalytic ability completely but has mutation-specific, altered kinetic properties, such as a different $K_{m}$ or $V_{\text {max }}$. Storage of glycosphingolipids occurs only if the velocity of the substrate load into the lysosome is greater than the velocity of degradation $\left(V_{\max }\right)$ by the corresponding enzyme. ${ }^{21}$ In milder forms of sphingolipidoses, there is sufficient residual enzyme activity so that the accumulation of storage occurs only at a very slow rate. This model could be verified in vitro in lysosomal storage disorders such as $\mathrm{G}_{\mathrm{M} 2}$-gangliosidosis, MLD, ${ }^{22}$ and Gaucher disease. ${ }^{23}$

Biomarkers, which reflect the activity of a disease process, ${ }^{24}$ can be diagnostic, prognostic, predictive, or pharmacodynamic. ${ }^{25}$ For a biomarker to be considered a true surrogate marker, it must (i) capture the severity of disease and (ii) reflect the net effect of treatment on the true outcome. ${ }^{26}$ It can be challenging to demonstrate this relationship in a rare disease. ${ }^{27}$

Drug development for MPS VII is challenging because the condition is ultrarare. Approval may become difficult if a neurological end point is required; however, there may be a higher chance of success because there is a regulatory precedent in similar conditions. ${ }^{28}$

\section{Limitations and directions for future research}

This study has several limitations. The survival data are, at least in part, mathematical estimations and, in part, historical. The standard of supportive care may have changed over time, which could not be quantified in this type of analysis. Of interest, survival rates between patients published before and after 1990 did not differ, which may mean that the impact of improved supportive care over time may have only a small effect size. Given the rare nature of disease, a prospective natural history study to obtain survival data would require many years to complete, and it would be difficult to recruit in an international multicenter setting. Because specific therapies are being developed, the present data are timely and of value. A common problem in orphan drug development is that natural history data are often elaborated only when therapy becomes available, which renders natural history studies-especially if survival is a pivotal end point-essentially infeasible. Therefore, the present study focused on hard end points, such as survival. Softer end points of the clinical phenotype, such as cognitive development, longitudinal variation of biomarkers, and physical features, as well as concomitant medication and supportive care, are best studied prospectively with a standardized protocol to avoid ascertainment bias and missing data. ${ }^{8,29}$ In addition, confounders for the survival outcome, such as surgeries and other supportive care measures, that remain unknown in this study are of particular interest for future research. Interviews and chart reviews, such as the work by Montaño and colleagues, ${ }^{6}$ are other options for obtaining timely, informative insight, although this approach is not free from the previously mentioned bias. Enzyme assays and GAG measurements in the present analysis were obtained in different laboratories and pooled; analytical methods in the respective laboratories may vary and may have changed over time. This may contribute to some statistical noise. In addition, source documents were not monitored. This analysis assumes that the published data are correct and accurate. Ideally, for 
future studies, specialty laboratory parameters should be conducted in a central laboratory if logistical issues in worldwide multicenter rare-disease studies are manageable. Cutoff values were computed with a relative new and widely used recursive partition technique. Because of the historical nature of the analyzed data, further prospective studies should evaluate the cutoff values or impact of different statistical methods.

The present study defines survival, diagnostic delay, and phenotype-biomarker association for MPS VII. These data will inform future clinical trials and serve as a baseline for future medical progress. The method of this research work-the definition of hard and quantitative clinical end points from published clinical data-exemplifies that important knowledge gaps in ultra-orphan diseases can be closed in a timely manner.

\section{Conclusion}

MPS VII is a disease with prenatal onset. In the absence of a prenatal diagnosis, most cases become clinically apparent at birth. MPS VII is an important differential diagnosis of nonimmune hydrops fetalis. There is considerable diagnostic delay. Our data corroborate a phenotype-biomarker association in MPS VII: patients with residual GUS activity in fibroblasts $>1.4 \%$ of normal controls had later onset of disease and lived longer than patients with GUS enzyme activities below this threshold. Patients with urinary GAG excretion $<602 \%$ of normal controls had longer survival than patient with higher levels of urinary GAG excretions. The survival data will help in future natural history and therapeutic studies.

\section{SUPPLEMENTARY MATERIAL}

Supplementary material is linked to the online version of the paper at http://www.nature.com/gim

\section{ACKNOWLEDGMENTS}

M.Z. received support from the Physician-Scientist Program at Ruprecht-Karls-University Heidelberg Faculty of Medicine. No funding was secured for this study.

\section{DISCLOSURE}

M.R. received consultancy fees or research grants from Alexion, GSK, Oxyrane, and Shire. The other authors declare no conflict of interest.

\section{REFERENCES}

1. Sly WS, Quinton BA, McAlister WH, Rimoin DL. Beta glucuronidase deficiency: report of clinical, radiologic, and biochemical features of a new mucopolysaccharidosis. J Pediatr 1973;82:249-257.

2. Tomatsu S, Fukuda S, Sukegawa K, et al. Mucopolysaccharidosis type VII: characterization of mutations and molecular heterogeneity. Am J Hum Genet 1991:48:89-96.

3. Human Gene Mutation Database. GUSB entry. 2016. http://www.hgmd.cf.ac. uk/ac/gene.php?gene=GUSB. Accessed 18 August 2016.

4. Orphanet. Orphanet Rare Diseases Collection. Prevalence distribution of rare diseases: bibliographic data. November 2016. http://www.orpha.net/ orphacom/cahiers/docs/GB/Prevalence_of_rare_diseases_by_alphabetical_list. pdf. Accessed 8 March 2017.

5. US Food and Drug Administration. Orphan Drug Designations and Approvals. 2016. https://www.accessdata.fda.gov/scripts/opdlisting/oopd/. Accessed 17 August 2016.
6. Montaño AM, Lock-Hock N, Steiner RD, et al. Clinical course of sly syndrome (mucopolysaccharidosis type VII). J Med Genet 2016;53:403-418.

7. Sands MS. Mucopolysaccharidosis type VII: A powerful experimental system and therapeutic challenge. Pediatr Endocrinol Rev 2014;12(suppl 1):159-165.

8. Mechler K, Mountford WK, Hoffmann GF, Ries M. Ultra-orphan diseases: a quantitative analysis of the natural history of molybdenum cofactor deficiency. Genet Med 2015;17:965-970.

9. Hothorn T, Hornik K, Zeileis A. Unbiased recursive partitioning: a conditional inference framework. J Comput Graph Stat 2006;15:651-674.

10. Gimovsky AC, Luzi P, Berghella V. Lysosomal storage disease as an etiology of nonimmune hydrops. Am J Obstet Gynecol 2015;212:281-290.

11. Huynh HT, Grubb JH, Vogler C, Sly WS. Biochemical evidence for superior correction of neuronal storage by chemically modified enzyme in murine mucopolysaccharidosis VII. Proc Natl Acad Sci USA 2012;109:17022-17027.

12. Rowan DJ, Tomatsu S, Grubb JH, et al. Long circulating enzyme replacement therapy rescues bone pathology in mucopolysaccharidosis VII murine model. Mol Genet Metab 2012;107:161-172.

13. Jackson M, Derrick Roberts A, Martin E, Rout-Pitt N, Gronthos S, Byers S. Mucopolysaccharidosis enzyme production by bone marrow and dental pulp derived human mesenchymal stem cells. Mol Genet Metab 2015;114:584-593.

14. Gurda BL, De Guilhem De Lataillade A, Bell P, et al. Evaluation of AAVmediated gene therapy for central nervous system disease in canine mucopolysaccharidosis VII. Mol Ther 2016;24:206-216.

15. Hofling AA, Devine S, Vogler C, Sands MS. Human CD34+ hematopoietic progenitor cell-directed lentiviral-mediated gene therapy in a xenotransplantation model of lysosomal storage disease. Mol Ther 2004;9:856865.

16. Derrick-Roberts AL, Pyragius CE, Kaidonis XM, Jackson MR, Anson DS, Byers $S$. Lentiviral-mediated gene therapy results in sustained expression of $\beta$-glucuronidase for up to 12 months in the gus $(\mathrm{mps} / \mathrm{mps}$ ) and up to 18 months in the gus(tm(L175F)Sly) mouse models of mucopolysaccharidosis type VII. Hum Gene Ther 2014;25:798-810.

17. Fox JE, Volpe L, Bullaro J, Kakkis ED, Sly WS. First human treatment with investigational rhGUS enzyme replacement therapy in an advanced stage MPS VII patient. Mol Genet Metab 2015;114:203-208.

18. Ries M. Biochemical Markers of Lipid Storage and Vasculopathy in Fabry Disease. Mathematisch-Naturwissenschaftliche Fakultät, Rheinische FriedrichWilhems-Universität Bonn: Bonn, Germany, 2008.

19. Ries M, Gal A. Genotype-phenotype correlation in Fabry disease. In: Mehta A, Beck M, Sunder-Plassmann G (eds). Fabry Disease: Perspectives from 5 Years of FOS. Oxford PharmaGenesis: Oxford, UK, 2006.

20. Goker-Alpan O, Hruska KS, Orvisky E, et al. Divergent phenotypes in Gaucher disease implicate the role of modifiers. J Med Genet 2005;42:e37.

21. Conzelmann E, Sandhoff K. Partial enzyme deficiencies: residual activities and the development of neurological disorders. Dev Neurosci 1983;6:58-71.

22. Leinekugel $P$, Michel S, Conzelmann E, Sandhoff K. Quantitative correlation between the residual activity of beta-hexosaminidase $\mathrm{A}$ and arylsulfatase $\mathrm{A}$ and the severity of the resulting lysosomal storage disease. Hum Genet 1992;88:513-523.

23. Schueler UH, Kolter T, Kaneski CR, Zirzow GC, Sandhoff K, Brady RO. Correlation between enzyme activity and substrate storage in a cell culture model system for Gaucher disease. J Inherit Metab Dis 2004;27: 649-658.

24. Katz R. Biomarkers and surrogate markers: an FDA perspective. NeuroRx 2004;1:189-195

25. US Food and Drug Administration. Center for Drug Evaluation and Research. Guidance for industry: qualification process for drug development tools. 2014. http://www.fda.gov/downloads/Drugs/ GuidanceComplianceRegulatoryInformation/Guidances/UCM230597.pdf. Accessed 30 September 2016.

26. Prentice RL. Surrogate endpoints in clinical trials: definition and operational criteria. Stat Med 1989;8:431-440.

27. Schiffmann R, Ries M, Blankenship D, et al. Changes in plasma and urine globotriaosylceramide levels do not predict Fabry disease progression over 1 year of agalsidase alfa. Genet Med 2013;15:983-989.

28. Mechler K, Mountford WK, Hoffmann GF, Ries M. Pressure for drug development in lysosomal storage disorders - a quantitative analysis thirty years beyond the US orphan drug act. Orphanet J Rare Dis 2015;10:46.

29. Mehta A, Beck M, Elliott P, et al.; Fabry Outcome Survey investigators. Enzyme replacement therapy with agalsidase alfa in patients with Fabry's disease: an analysis of registry data. Lancet 2009;374:1986-1996. 